\title{
Assessment of pain in patients with juvenile rheumatoid arthritis: relation between pain intensity and degree of joint inflammation
}

\author{
Norman T Ilowite, Gary A Walco, Rubem Pochaczevsky
}

\begin{abstract}
The relation between pain and joint inflammation in patients with juvenile rheumatoid arthritis has not previously been systematically evaluated. Eighteen patients with juvenile rheumatoid arthritis completed paediatric pain questionnaires and the joints affected were examined by thermography. Although significant correlations were shown between parent and doctor pain intensity ratings and joint temperature, correlations of patient pain intensity ratings and joint temperature were only significant in younger children. The degree of joint inflammation is only one factor of several contributing to the amount of subjective pain experienced by children with juvenile rheumatoid arthritis, indicating the need for a comprehensive assessment of the relatively independent variables of inflammation and pain in children with juvenile rheumatoid arthritis.
\end{abstract}

Pain is a major problem in children with juvenile rheumatoid arthritis and is an important factor in their general health status. ${ }^{1}$ Effective interventions require a systematic evaluation of pain, including a reliable means of assessing the intensity of subjective pain and a measure of the potential peripheral stimulation of pain receptors (nociceptive stimulation). Nociceptive stimulation, in juvenile rheumatoid arthritis, is presumed to be caused by tissue damage secondary to joint inflammation. The present study aims at testing the relation between patients' reports of subjective pain and the degree of joint inflammation.

Previous studies have shown the reliability, validity, and clinical sensitivity of visual analogue scales (VAS) as a measure of the intensity of subjective pain in children with chronic or recurrent pain, including joint pain. ${ }^{2}{ }^{3} \mathrm{McGrath}{ }^{4}$ described calibration studies which indicate that children's VAS scores are rational in nature-that is, there is a direct arithmetic relation between VAS scores and perceptual changes. Verbal descriptors have also been used to assess the sensory, affective, and evaluative aspects of children's experience of pain. ${ }^{2}$

The quantitative relation between joint inflammation and nociceptive stimulation is unclear. Inflammation in the joint results in the disruption of cellular membranes, freeing lipid components of the membrane to be acted upon by phospholipase A, converting them to arachidonic acid. Arachidonic acid is further acted upon by cyclooxygenase to enter the prosta- glandin cascade, resulting in the formation of prostaglandins $\mathrm{PGE}_{2}$ and $\mathrm{PGI}_{2}$. These prostaglandins sensitise pain receptors (nociceptors) to the stimulatory actions of bradykinins and histamines, chemical mediators which are also released into the cellular microenvironment as a consequence of joint inflammation. ${ }^{5}$ There is no ideal measure of joint inflammation in juvenile rheumatoid arthritis. Laboratory parameters, such as erythrocyte sedimentation rate, do not correlate well with the degree of joint inflammation. ${ }^{6}$ Assessments by doctors of joint inflammation are based on laboratory results and physical findings such as the degree of swelling, warmth, and limitation of motion in the affected joints. The latter measures are subjective and may be further biased by observations of the patients' behaviour towards pain. Therefore, this putative objective measure of joint inflammation may not serve as an independent variable when trying to determine the relation between inflammation and subjective pain.

Although not an ideal measure of joint inflammation, joint temperature, as measured by thermography of skin overlying the affected joints, has been shown to be a reasonable measure of disease activity and inflammation in rheumatoid arthritis and juvenile rheumatoid arthritis. ${ }^{7-9}$ Thermographic results correlate well with the duration of morning stiffness, a number of articular index scores, and parallel changes in these parameters after treatment with anti-inflammatory drugs. ${ }^{7-9}$

Thus, to determine the relation between joint inflammation and subjective pain, correlations between the temperature of the skin overlying the joint, an objective index of joint inflammation, and child, parent, and doctor reports of pain intensity were studied.

\section{Patients and methods}

PATIENTS

The patients were three boys and 15 girls between the ages of 4.7 and 16.7 years (mean 9.6 (range 4-17) years), fulfilling the American College of Rheumatology criteria for the diagnosis of juvenile rheumatoid arthritis. ${ }^{10}$ Eleven patients had pauciarticular arthritis, five had polyarticular arthritis, and two had systemic onset disease. This representation of subtypes of juvenile rheumatoid arthritis approximates their relative incidence in large series of patients with juvenile rheumatoid arthritis. ${ }^{11}$

The paediatric pain questionnaire, with parent, 
child, and adolescent forms, includes a number of modalities aimed at assessing various components of pain in paediatric patients. ${ }^{12}$ Principally used in this study were visual analogue scales, which are $10 \mathrm{~cm}$ lines with no marks along them, anchored with the words 'no pain', 'not hurting', and 'no discomfort' on one end, and 'hurting a whole lot', 'very uncomfortable', and 'severe pain' on the other. Children and parents were simply instructed to place a mark along the line at a level representing the intensity of their present pain. The attending paediatric rheumatologist (NTI) also completed a VAS immediately after a physical examination. In addition, the paediatric pain questionnaire includes a list of $\mathbf{4 6}$ pain descriptors reflecting the sensory, affective, and evaluative aspects of pain. We wished to determine the relation of endorsed adjectives related to thermal sensations ('burning', 'hot', 'warm', 'cool', 'cold') and the difference in temperature between the affected joints and contralateral joints or between skin overlying the joint and adjacent non-articular tissue.

\section{THERMOGRAPHY}

Liquid crystals used for thermography are cholesterol derivatives that selectively reflect polarised light in a narrow region of wavelengths. ${ }^{13} 14$ They have strong molecular rotatory power and the ability to change colour in response to temperature variations. These changes may be shown graphically by contact colour thermography. The crystals are embedded in elastic sheets (Flexi-Therm, Westbury, NY, USA) which, when inflated, conform to body surfaces. These resultant colour changes are permanently recorded by an attached camera, and have been shown to be reliable to $0: 1^{\circ} \mathrm{C}$. The lowest temperature is displayed as dark brown, with progressive temperature increases shown as reddish brown, yellow, green, light blue, and dark blue. Colours have been calibrated so that a specific temperature value can be derived. A delta T (dT) score was calculated on the basis of the difference in temperature between the affected and contralateral joints $\left(\mathrm{dT}_{\text {con }}\right)$, for asymmetric disease, or between the skin overlying the joint and adjacent nonarticular tissue ( $\mathrm{dT}_{\mathrm{adj}}$ ), for symmetric arthritis. The highest observed $d T$ score was chosen for analysis.

\section{STATISTICAL ANALYSES}

VAS and dT scores represent rational data. As a result, Pearson product moment correlations were performed to assess the relation between pain intensity (VAS scores) and joint inflammation (dT values). The method of least squares was used to determine regression lines indicating the relation between dT values and VAS scores. The relation between endorsed pain descriptors reflecting thermal sensations and $d T$ values was assessed with $t$ tests.

\section{Results}

The mean (SD) number of joints affected was
$4.5(5 \cdot 76)$ (range zero to greater than 20$)$. $\mathrm{dT}_{\text {con }}$ values ranged from 0 to $3 \cdot 1^{\circ} \mathrm{C}$ (mean (SD) $1 \cdot 81$ $(0.97)) ; \mathrm{dT}_{\text {adj }}$ values ranged from 0 to $3.0^{\circ} \mathrm{C}$ (mean (SD) $1.24(0 \cdot 97)$ ). The highest dT value was used for comparison with VAS scores; in nine patients the $\mathrm{dT}_{\text {con }}$ value was equal to the $\mathrm{dT}_{\mathrm{adj}}$; in two patients the $\mathrm{dT}_{\text {con }}$ was higher; and in seven patients the $d T_{\text {adj }}$ was higher. VAS scores potentially range from 0 to $10 \mathrm{~cm}$. VAS scores determined by the children covered the entire range (mean (SD) $2 \cdot 44(3 \cdot 28)$ ), VAS scores determined by the parents ranged from 0 to $8 \cdot 1$ (mean (SD) $2 \cdot 18(2 \cdot 48)$ ), and VAS scores determined by the doctor ranged from 0 to 9.5 (mean (SD) 4.86 (2.97)).

Figures 1 and 2 show that correlations between VAS scores determined by parents and doctors for present pain intensity, and dT values from thermography were significant $(\mathrm{r}=0.58, \mathrm{p}<0.01 ; \mathrm{r}=0.50, \mathrm{p}<0.05$, respectively). Figure 3 shows that this correlation for

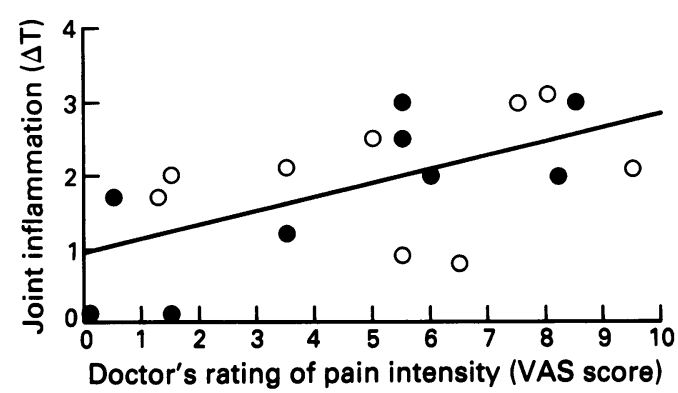

Figure 1 Relation between joint inflammation and doctor's assessment of pain intensity, with regression line. Values for younger patients are shown by open symbols; those for older patients by closed symbols (see text).

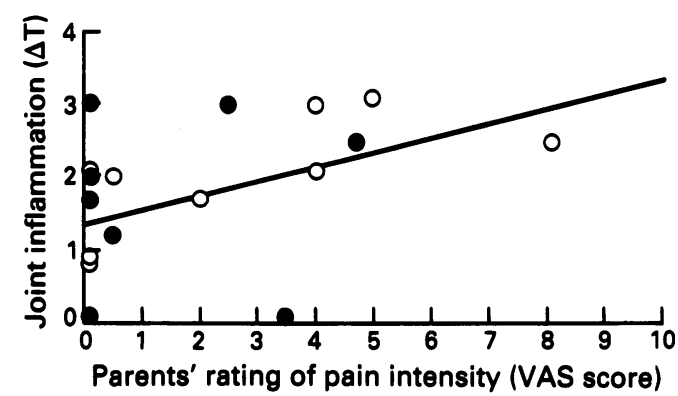

Figure 2 Relation between joint inflammation and assessment of pain intensity by parents, with regression line. $V$ alues for younger patients are shown by open symbols; those for older patients by closed symbols (see text).

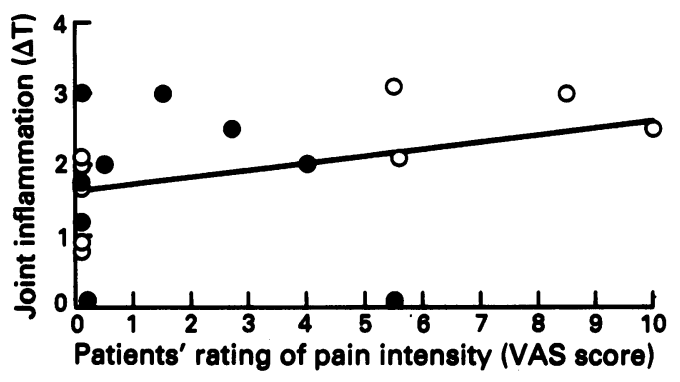

Figure 3 Relation between joint inflammation and assessment of pain intensity by patients, with regression line. Values for younger patients are shown by open symbols; those for older patients by closed symbols (see text). 
children's ratings of their pain intensity was not statistically significant $(r=0.32, p>0.05)$.

Although the cell sizes were sometimes small, further analyses were performed to examine the effects of age, disease subtype, and the number of joints affected. It was noted that for patients younger than $7 \cdot 86$ years (median split), the correlation between dT values and patient VAS scores was $(r=0.73, p<0.05)$, whereas for older patients this value was much lower $(r=-0 \cdot 24$, p>0.05) (fig 3). Analyses for VAS scores determined by parents yielded a similar discrepancy betwen younger and older patients $(\mathrm{r}=0.70, \mathrm{p}<0.05 ; \mathrm{r}=0.24, \mathrm{p}>0.05$, respectively) (fig 2). An opposite trend was noted for the relation between VAS scores and $\mathrm{dT}$ values determined by the doctor, as for younger children the correlation was low $(r=0.26$, $p>0.05$ ), whereas for older children the correlation was significant $(r=0.77, p<0.01)$ (fig 1).

Analyses controlling for disease onset subtype indicated that in children with pauciarticular onset disease, VAS scores determined by parents correlated significantly with $\mathrm{dT}$ values $(r=0.63, p<0.05)$. Cell sizes among children with either polyarticular or systemic onset disease were too small to conduct meaningful analyses. No differences were observed in the correlations between VAS scores and $\mathrm{dT}$ values as a function of the number of affected joints (less than or equal to, versus greater than four).

Analyses ( $t$ tests) of pain descriptors showed no significant differences in dT values between children who chose adjectives reflecting heat ('burning', 'hot', 'warm') and those who did not. Descriptors reflecting lack of heat ('cool' and 'cold') were each endorsed by only one patient, which made systematic analyses impossible.

\section{Discussion}

Pain in children is best understood as a multifactorial concept. Comprehensive assessment of paediatric pain includes the child's subjective experience, behavioural manifestations, physiological parameters, and relevant environmental factors. ${ }^{4}$ The significant correlations between increased temperature in the affected joints and pain intensity ratings by parents and the doctor suggests that there is a strong relation between the degree of joint inflammation and pain. Specifically, as found in previous studies, it is likely that parents and doctors rely on behavioural cues from the child in determining the level of subjective pain intensity. ${ }^{3}$ In addition, because the joint temperature can be estimated by touch, parents and doctors may be inferring discomfort as a function of observed joint temperature.

This correlation was not nearly as strong for children's reports of subjective pain intensity. These findings suggest that the relation between joint inflammation and pain intensity is not direct, as only $10 \%$ of the variance was accounted for. Furthermore, analyses of pain descriptors indicated no significant differences in joint inflammation between children who chose adjectives reflecting heat ('burning', 'hot', 'warm') and those who did not. Therefore, it is likely that several interrelated factors combine to determine a given child's subjective pain experience, inflammation being only one of these factors.

Disease severity has been shown to account for a relatively small percentage of the variance among factors determining the subjective pain intensity of patients with juvenile rheumatoid arthritis. ${ }^{12}$ Indeed, in this study, neither disease type nor number of joints affected was a significant factor in differentiating the relation between dT values and VAS scores. Other psychological factors, such as developmental level, must also be considered. ${ }^{15} 16$ These data indicate that in younger children, the relation between joint inflammation and perceived level of pain intensity was fairly strong as the dT values accounted for $53 \%$ of the variance in the children's VAS scores. In contrast, in older children, the correlation was much lower and negative. This lack of a relation may be due to the fact that in older children, many more socioemotional factors affect the perception of pain. Although based on very different findings, Beales $e t a l^{17}$ also suggested that emotional factors play a more important role in pain perception in older patients with juvenile rheumatoid arthritis.

Other workers have reported a lack of association between inflammation of the joint and reports of subjective pain in some patients with juvenile rheumatoid arthritis. ${ }^{18}$ Fourteen per cent of patients with juvenile rheumatoid arthritis, especially young patients with pauciarticular disease, did not report the presence of pain, despite physical evidence of joint inflammation. However, pain was not systematically evaluated in the children, which is especially important in young children, who often do not report pain.

It was interesting to note that in some of the younger patients, for whom colours are used on body outlines as a means of identifying painful sites and relative levels of intensity, the degree of pain matched thermographic findings. For example, one 5 year old girl coloured her right knee in red (a colour she previously defined as representing the most intense pain) and her left ankle in blue (the colour she chose as moderate pain). Thermographic data indicated that the affected knee was warmer than the ankle. Systematic analyses failed to yield any clear relation between the colours chosen and joint inflammation. Thus, colours should be used as an individual means of expressing relative pain levels without previous assumptions about the significance of a given colour.

There is an issue which may confound the data and must be considered. In other disease states, notably reflex sympathetic dystrophy and nerve root syndromes, the skin temperature of the affected hands and feet decreases in relation to increased reports of pain. ${ }^{131419}$ Therefore, if the effects of joint inflammation could be theoretically separated from pain, it is possible that arthritis pain may decrease the skin temperature compared with effects of joint inflammation alone.

Beyond theoretical interest, thermography, 
as a systematic means of assessing joint inflammation, has clinical importance. Current treatments for juvenile rheumatoid arthritis focus principally on controlling inflammation and these data show that reported pain alone is not an ideal index of inflammation. Therefore, thermography is a reasonable dependent measure of interventions aimed at reducing inflammation.

Reducing inflammation should not be the only goal of treatment, however. In the comprehensive care of children with juvenile rheumatoid arthritis, multiple strategies should be used to reduce pain and increase levels of adaptive functioning. This may include pharmacological interventions aimed at reducing inflammation, physical treatment to improve musculoskeletal functioning, and cognitive behavioural techniques to alter the experience of pain and enhance adaptive functioning. ${ }^{20}$ Systematic evaluation of pain and joint inflammation is a critical first step in realising this goal.

This work was in part supported by a grant from the Easter Seal Research Foundation. The authors acknowledge Lauren Riordan, MA, for her assistance with data collection and coding.

1 Lovell D J, Walco G A. Pain associated with juvenile rheumatoid arthritis. Pediatr Clin North Am 1989; 36: 1015-27.

2 Varni J W, Thompson K L, Hanson V. The Varni/Thompson pediatric pain questionnaire: I. Chronic musculoskeletal

pain in juvenile rheumatoid arthritis. Pain 1987; 28: 27-38.

Walco G A, Dampier C D. Pain in children and adolescents with sickle cell disease: a descriptive study. $\mathcal{F}$ Pediat Psychol 1990; 15: 643-58.

4 McGrath P A. Pain in children: nature, assessment, and treatment. New York: Guilford Press, 1990.
5 Kantor T G. Concepts in pain control. Semin Arthritis Rheum 1989; 18: 94-9.

6 Giannini E H, Brewer E T. Poor correlation of the erythrocyte sedimentation rate and clinical activity in juvenile rheumasedimentation rate and clinical activity in juvenile
toid arthritis. Clin Rheumatol 1987; 6: 197-201.

7 Devereaux M D, Parr G R, Thomas D P, Hazleman B L. Disease activity indexes in rheumatoid arthritis; a prospective, comparative study with thermography. Ann Rheum Dis 1985; 44: 434-7.

8 Bacon P A, Davies J, Ring E F J. Benoxaprofen-dose-range studies using quantitative thermography. $f$ Rheumatol Studies using quantitati $1980 ; 6$ : 48-53.

9 Mignone F, Sardella $M$, Barbero I, et al. Application of thermography in the diagnosis and follow-up of juvenile rheumatoid arthritis. Minerva Pediatr 1986; 38: 231-4.

10 Brewer E J, Bass J, Baum J, et al. Current proposed revision of JRA criteria. Arthritis Rheum 1977; 20: 195-9.

11 Schaller J G. Juvenile rheumatoid arthritis. Series 1. Arthritis Rheum 1977; 20 (suppl): 165-70.

12 Walco G A, Varni J W. Chronic and recurrent pain: hemophilia, JRA and sickle cell disease. In: Bush J P, Harkins S W, eds. Children in pain: clinical and research issues from a developmental perspective. New York: Springer, 1990.

13 Pochaczevsky R, Wexler C E, Meyers P H, Epstein J A, Marc $J$ A. Liquid crystal thermography of the spine and extremities: its value in the diagnosis of spinal root syndromes. $f$ Neurosurg 1982; 56: 386-95.

14 Pochaczevsky $R$. Thermography in skeletal and soft tissue trauma. In: Tavaras J M, Ferrucci F, Norman A, eds. Radiology: diagnostic imaging and intervention. Vol 5. Philadelphia: Lippincott, 1987: 1-7.

15 Varni J W, Wilcox K T, Hanson V, Brik R. Chronic musculoskeletal pain and functional status in juvenile rheumatoid arthritis: an empirical model. Pain 1988; 32: rheum.

16 Thompson K L, Varni J W. A developmental cognitivebiobehavioural approach to pediatric pain assessment. Pain 1986; 25: 283-96.

17 Beales J G, Keen J H, Holt P J L. The child's perception of the disease and experience of pain in juvenile chronic arthritis. F Rheumatol 1983; 10: 61-5.

18 Sherry D D, Bohnsack J, Salmonson K, Wallace C A, Mellins E. Painless juvenile rheumatoid arthritis. $\mathcal{F}$ Pediatr 1990; 116: 921-3.

19 Lightman H I, Pochaczevsky R, Aprin H, Ilowite N T. Thermography in childhood reflex sympathetic dystrophy. f Pediatr 1987; 111: 551-5.

20 Walco G A, Varni J W, Ilowite N T. Cognitive-behavioral pain management in children with juvenile rheumatoid arthritis. Pediatrics. In press. 